\title{
Role of peroxiredoxin III in the pathogenesis of pre-eclampsia as evidenced in mice
}

\author{
Lianqin Li, ${ }^{1, *}$ Masuo Obinata ${ }^{2}$ and Katsuyoshi Hori ${ }^{3}$ \\ 'Obstetrics and Gynecology Center; Tsinghua University Second Hospital; Beijing, China \\ ${ }^{2}$ Department of Cell Biology; and ${ }^{3}$ Department of Vascular Biology; Institute of Development, Aging and Cancer; Tohoku University; Sendai, Japan
}

Key words: peroxiredoxin, placenta, oxidative stress, hypertension, pre-eclampsia, mouse, reactive oxygen species

Abbreviations: ROS, reactive oxygen species; Prx, peroxiredoxin; PE, preeclampsia; TNF $\alpha$, tumor necrosis factor alpha; ET-1, endothelin-1

Submitted: 09/10/09

Revised: 09/15/09

Accepted: 09/16/09

Previously published online: www.landesbioscience.com/journals/ oximed/article/10094

* Correspondence to:

Lianqin Li; Email: lilq2005@I26.com

\begin{abstract}
As a member of peroxiredoxin (Prx) Afamily, PrxIII has been demonstrated to play an important role in scavenging intracellular reactive oxygen species (ROS). Since PrxIII knockout mice exhibited oxidative stress in placentas resembling pathophysiologic changes in placentas of human pre-eclampsia, we measured blood pressure through the carotid artery and detected oxidative status by western blotting in pregnant mice. We did not notice hypertension in pregnant PrxIII knockout mice as compared with wild-type littermates, although endothelin-1 was overexpressed in PrxIII-deficient placentas. Our results indicate that PrxIII is not involved in pre-eclamptic development. Instead, PrxIII is an indispensable antioxidant in placentas where oxidative stress exists.
\end{abstract}

\section{Introduction}

As a unique organ that connects mother and fetus, the placenta provides the place for materno-fetal exchange of gases, nutrients and metabolic products. Since placenta is abundant in mitochondria, large amount of reactive oxygen species (ROS) is continuously produced during metabolic processes. During pregnancy, ROS production is gradually elevated with the increase of energy demand due to both fetal and maternal requirements. Although small amount of ROS is considered to be a second messenger, excessive ROS would cause oxidative injuries on DNA, lipids, and proteins. Organisms have developed multiple antioxidant defenses against oxidative damage. For example, placental antioxidant activity was increased with gestation progression, ${ }^{1}$ and higher activity of superoxide dismutase was observed in maternal erythrocytes. ${ }^{2}$ More importantly, mitochondrion possesses a primary antioxidant chain that is composed of peroxiredoxin III (PrxIII), thioredoxin II and thioredoxin reductase. ${ }^{3}$

As a member of peroxiredoxin family, PrxIII was found to be responsible to oxidative stress in placentas. ${ }^{4,5} \mathrm{We}$ have recently observed placental oxidative damage in PrxIII knockout (PrxIII ${ }^{-/}$) mice. ${ }^{6}$ Interestingly, our findings in PrxIII-deficient placentas were quite similar to the major pathophysiologic changes in placentas of pre-eclampsia $(\mathrm{PE}),{ }^{7-9}$ a serious pregnancy complication in human beings. Since it is speculated that increased placental lipid peroxides and/or tumor necrosis factor alpha (TNFalpha) cause activation of leukocytes which serve as circulating mediators and contribute to endothelial dysfunction and subsequent hypertension, ${ }^{10}$ we have investigated whether placental oxidative damage causes hypertensive disorders in mice resembling PE.

\section{Results}

Blood pressure in pregnant mice. Since oxidative damage in PrxIII-deficient placentas of mice resembled pathological findings in pre-eclamptic placentas, ${ }^{6-9}$ we measured mean arterial blood pressure in pregnant mice trying to elucidate pathogenetic role of PrxIII in pre-eclamptic development. As indicated in Table 1, there was no significant difference of blood pressure 
Table I. Blood pressure in PrxIII ${ }^{+/+}$, PrxIII+/- and Prxlll--/ mice before and after pregnancy

$\begin{array}{cccc}\text { Blood Pressure } & \text { PrxIII+/+ } & \text { PrxIII } & \text { PrxIII-/- }^{+/-} \\ \text {Before Pregnancy } & 95 \pm 2 & 95 \pm 2 & 95 \pm 3 \\ \text { After Pregnancy } & 95 \pm 6 & 93 \pm 5 & 97 \pm 5\end{array}$

Blood pressure in mice was measured as described in "Materials and methods" section ( $n=10$ for $\mathrm{PrxlII}^{+/+}$, PrxIII ${ }^{+/-}$and PrxIII-/- mice respectively). There was no significant difference of blood pressure among individual groups before or after pregnancy $(p>0.05)$.

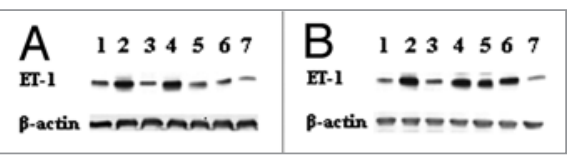

Figure I. Western blotting analysis for ET-I expression in $\operatorname{Prx} \mathrm{II}^{+/+}(\mathrm{A})$ and $\mathrm{Pr} x \mathrm{III}^{-/-}(\mathrm{B})$ mouse organs including (from I to 7) brain, heart, lung, liver, kidney, placenta and skeletal muscle. Signals for ET-I protein were significantly enhanced in Prxlll knockout placenta and kidney as compared with wild-type organs.

before or after pregnancy among PrxIII ${ }^{+/+}$, PrxIII ${ }^{+/-}$and PrxIII ${ }^{-/-}$mice.

Systemic oxidative status in pregnant mice. To further understand oxidative status in pregnant mice, we detected expression of endothelin-1 (ET-1) systemically. As shown in Figure 1, ET-1 protein was evidently enhanced in placentas derived from PrxIII ${ }^{--}$mice, which was in accordance with our previous report. ${ }^{6}$ Nevertheless, ET-1 expression was not significantly elevated in other PrxIII-deficient organs except kidney as compared with wild-type samples.

\section{Discussion}

We recently reported that PrxIII-deficiency resulted in excessive lipid peroxidation and TNFalpha production in placentas of mice, ${ }^{6}$ which was quite similar to the pathological changes in pre-eclamptic placentas. $^{7-9}$ We then measured blood pressure in pregnant mice and found serious oxidative stress in placentas of PrxIII knockout mice without subsequent dysfunction of peripheral endothelium and hypertensive disorder.

$\mathrm{PE}$ is one of the most important pregnancy complication characterized by hypertension, proteinuria, and edema. Although the precise mechanism for preeclamptic occurrence remains unclear, oxidative stress was considered to be one of the main causative factors. ${ }^{13,14}$ Malperfusion of the placenta was implicated as a cause of oxidative stress in complications of human pregnancy, ${ }^{15}$ and subsequent endothelial activation and peripheral vascular contraction was considered to be the basic pathophysiology of pre-eclampsia. ${ }^{16,17}$ Both TNFalpha and ET-1 were reported to play important roles in linking placental oxidative damage and systemic endothelial dysfunction in PE. ${ }^{17,18}$ We detected high levels of TNFalpha and ET-1 in PrxIII-deficient placentas of mice, implying serious oxidative stress and poor perfusion in the organs. Nevertheless, we did not notice the increase of ET-1 in other organs except kidney and subsequent hypertension in pregnant PrxIIImice. Combining with our results and another report that PrxIII is significantly upregulated in pre-eclamptic placentas, ${ }^{5}$ we suppose that PrxIII is not involved in the pathogenesis of pre-eclamptic development. Instead, it is very likely that PrxIII is an indispensable ROS scavenger in oxidation-threatened placentas and contributes to the inhibition of PE progression.

\section{Materials and Methods}

Animals. Experimental mice were generated by mating $\mathrm{C} 57 \mathrm{BL} / 6$ mice with PrxIII $^{-/-}$mice to generate PrxIII ${ }^{+/-}$offspring, which were then intercrossed to produce PrxIII ${ }^{+/+}$, PrxIII ${ }^{+/-}$and PrxIII ${ }^{-/}$littermates for experiments. PrxIII genotypes were determined by PCR analysis as previously described. ${ }^{11}$ Use of the experimental animals was approved by the institutional ethics committee of Tohoku University, Japan.

Measurement of blood pressure. Mean arterial blood pressure of mice was measured through the carotid artery. ${ }^{12}$ After anesthesia with diethyl ether the carotid artery of maternal mouse was isolated and a catheter was inserted into the artery through a tiny incision. Another end of the catheter was connected with a pressure transducer (Spectramed Medical Products, Singapore) whose output was fed into an amplifier (NEC-Sanei Co., Tokyo, Japan) and recorded. Age-matched non-pregnant female mice were used as controls.

Systemic detection of oxidative stress. Brains, hearts, lungs, livers, kidneys, skeletal muscles and placentas were removed from maternal mice that were pregnant for 18 days. Samples from age-matched non-pregnant mice were used as controls. The samples were homogenized through ultrasonic disruption and protein concentration was detected by Bio-Rad protein assay kit. After separation on 15\% SDSPAGE and transfer to Immobilon membrane (Millipore Corporation), proteins were recognized with primary antibody anti-ET-1 (Santa cruz). Visualization was achieved with ECL western blotting detection system (Amersham). Anti- $\beta$ actin antibody (Sigma) was used as internal control.

Statistic analysis. The value of blood pressure was presented as mean \pm SEM and was compared among PrxIII ${ }^{+/+}$, PrxIII ${ }^{+/-}$ and PrxIII ${ }^{-/}$mice by analysis of variance. $\mathrm{p}<0.05$ was considered to be statistically significant.

\section{References}

1. Qanungo S, Mukherjea M. Ontogenic profile of some antioxidants and lipid peroxidation in human placental and fetal tissues. Mol Cell Biochem 2000; 215:11-9.

2. Carone D, Loverro G, Greco P, Capuano F, Selvaggi L. Lipid peroxidation products and antioxidant enzymes in red blood cells during normal and diabetic pregnancy. Eur J Obstet Gynecol Reprod Biol 1993; 51:103-9.

3. Rabilloud T, Heller M, Rigobello MP, Bindoli A, Aebersold R, Lunardi J. The mitochondrial antioxidant defence system and its response to oxidative stress. Proteomics 2001; 1:1105-10.

4. Ejima K, Nanri H, Araki M, Koji T, Shibata E, Kashimura M, et al. Expression of mitochondrial thioredoxin-dependent antioxidant protein, SP-22, in normal human and inflammatory mouse placentae. Placenta 2000; 21:847-52.

5. Shibata E, Nanri H, Ejima K, Araki M, Fukuda J, Yoshimura K, et al. Enhancement of mitochondrial oxidative stress and upregulation of antioxidant protein peroxiredoxin III/SP-22 in the mitochondria of human pre-eclamptic placentae. Placenta 2003; 24:698-705.

6. Li L, Shoji W, Oshima H, Obinata M, Fukumoto M, Kanno N. Crucial role of peroxiredoxin III in placental antioxidant defense of mice. FEBS Lett 2008; 582:2431-4.

7. Wang Y, Walsh SW. TNFalpha concentrations and mRNA expression are increased in preeclamptic placentas. J Reprod Immunol 1996; 32:157-69. 
8. Morikawa S, Kurauchi O, Tanaka M, Yoneda M, Uchida K, Itakura A, et al. Increased mitochondrial damage by lipid peroxidation in trophoblast cells of preeclamptic placentas. Biochem Mol Biol Int 1997; 41:767-75.

9. Shibata E, Ejima K, Nanri H, Toki N, Koyama C, Ikeda M, et al. Enhanced protein levels of protein thiol/disulphide oxidoreductases in placentae from pre-eclamptic subjects. Placenta 2001; 22:566-72.

10. Gupta S, Agarwal A, Sharma RK. The role of placental oxidative stress and lipid peroxidation in preeclampsia. Obstet Gynecol Surv 2005; 60:807-16.

11. Li L, Shoji W, Takano H, Nishimura N, Aoki Y, Takahashi R, et al. Increased susceptibility of MER5 (peroxiredoxin III) knockout mice to lipopolysaccharide (LPS)-induced oxidative stress. Biochem Biophys Res Commun 2007; 355:715-21.
12. Hori K, Saito S. Microvascular mechanisms by which the combretastatin A-4 derivative AC7700 (AVE8062) induces tumour blood flow stasis. Br J Cancer 2003; 89:1334-44.

13. Roberts JM, Lain KY. Recent insights into the pathogenesis of pre-eclampsia. Placenta 2002; 23:359-72.

14. Hoffmann DS, Weydert CJ, Lazartigues E, Kutschke WJ, Kienzle MF, Leach JE, et al. Chronic tempol prevents hypertension, proteinuria and poor fetoplacental outcomes in $\mathrm{BPH} / 5$ mouse model of preeclampsia. Hypertension 2008; 51:1058-65.

15. Hung TH, Skepper JN, Charnock-Jones DS, Burton GJ. Hypoxia-reoxygenation: a potent inducer of apoptotic changes in the human placenta and possible etiological factor in preeclampsia. Circ Res 2002; 90:1274-81.
16. Hung TH, Skepper JN, Burton GJ. In vitro ischemiareperfusion injury in term human placenta as a model for oxidative stress in pathological pregnancies. Am J Pathol 2001; 159:1031-43.

17. Hung TH, Charnock-Jones DS, Skepper JN, Burton GJ. Secretion of tumor necrosis factor-alpha from human placental tissues induced by hypoxia-reoxygenation causes endothelial cell activation in vitro: a potential mediator of the inflammatory response in preeclampsia. Am J Pathol 2004; 164:1049-61.

18. Fiore G, Florio P, Micheli L, Nencini C, Rossi M, Cerretani D, et al. Endothelin-1 triggers placental oxidative stress pathways: putative role in preeclampsia. J Clin Endocrinol Metab 2005; 90:4205-10 


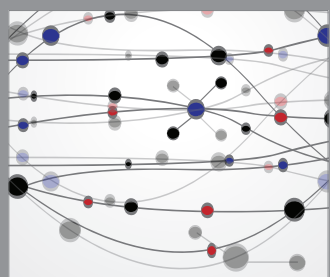

The Scientific World Journal
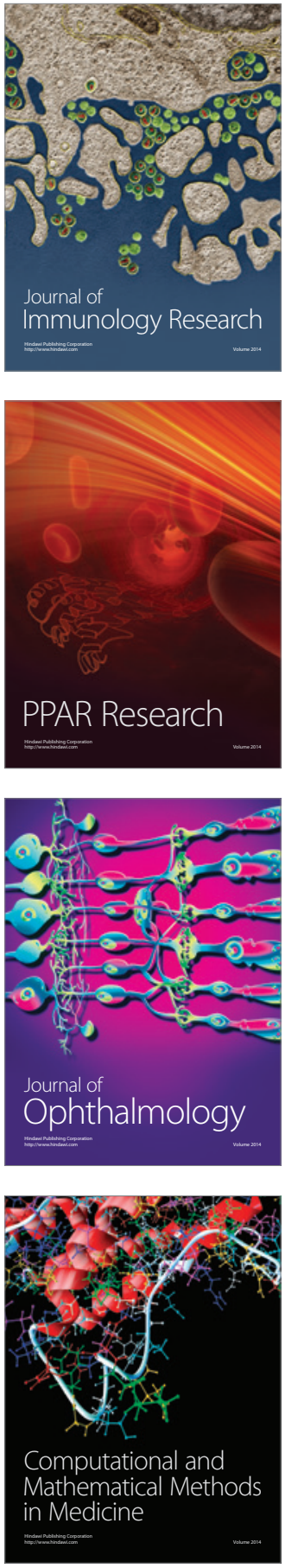

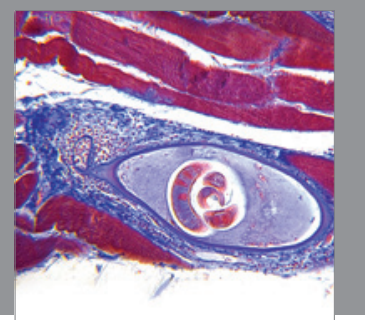

Gastroenterology

Research and Practice
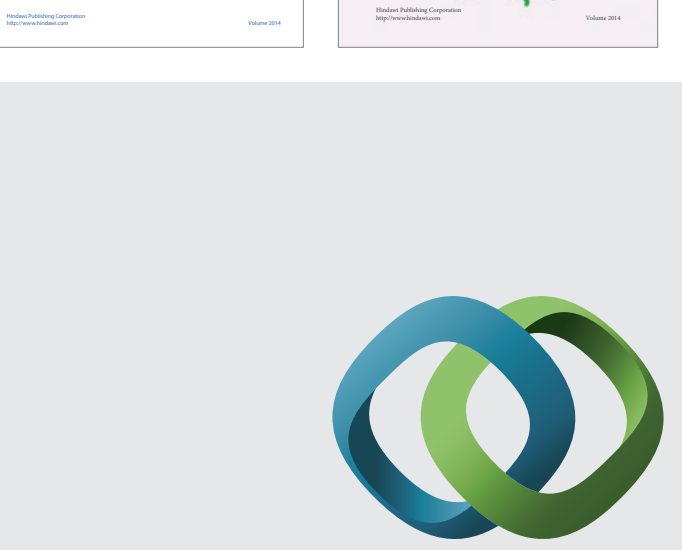

\section{Hindawi}

Submit your manuscripts at

http://www.hindawi.com
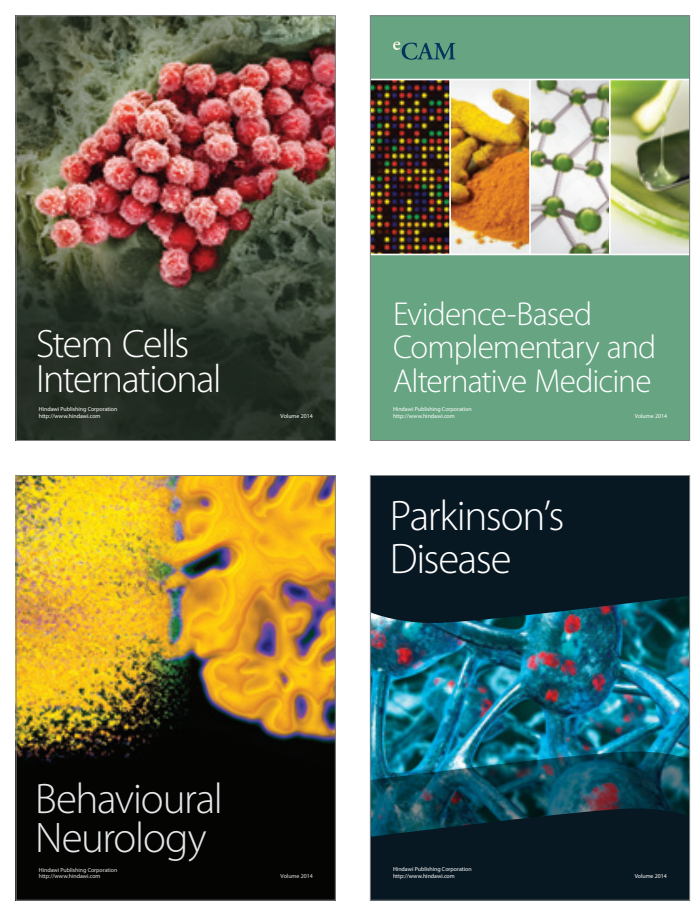

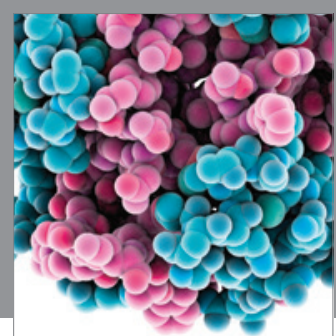

Journal of
Diabetes Research

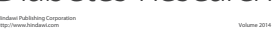

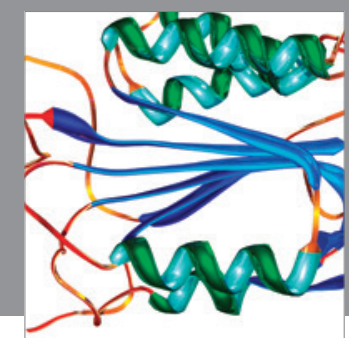

Disease Markers
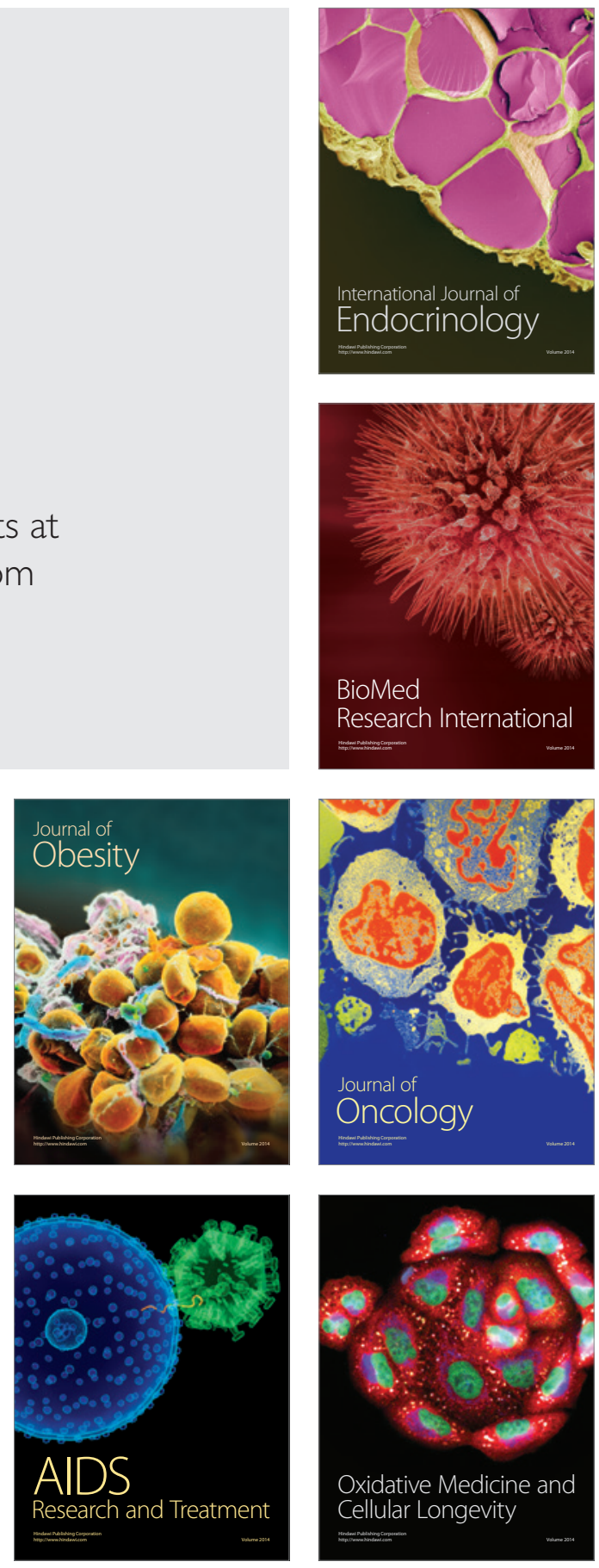